\title{
Conventional vs. pulsed-light accelerated corneal collagen cross-linking for the treatment of progressive keratoconus: 12-month results from a prospective study
}

\author{
LIANG-ZHU JIANG ${ }^{1,2}$, WEI JIANG ${ }^{3}$ and SHI-YAN QIU ${ }^{4}$ \\ ${ }^{1}$ Department of Ophthalmology, Shandong Provincial Hospital Affiliated to Shandong University, Jinan, Shandong 250000; \\ ${ }^{2}$ Department of Ophthalmology, The People's Hospital of Linyi, Linyi, Shandong 276000; ${ }^{3}$ Department of Rehabilitation, \\ The Affiliated Hospital of Southwest Medical University, Luzhou, Sichuan 646000; \\ ${ }^{4}$ Department of Pediatrics, The People's Hospital of Linyi, Linyi, Shandong 276000, P.R. China
}

Received October 21, 2016; Accepted June 8, 2017

DOI: 10.3892/etm.2017.5031

\begin{abstract}
The aim of the present study was to compare the clinical outcomes of conventional corneal collagen cross-linking (CXL) and pulsed-light accelerated CXL (pl-ACXL) in the eyes of patients with progressive keratoconus. A total of 72 eyes with progressive keratoconus in 58 patients were equally divided into the CXL and pl-ACXL treatment groups. The CXL treatment was performed using the UVX 1000 system with $0.1 \%$ riboflavin solution in $20 \%$ dextran presoak for $30 \mathrm{~min}$, and $3 \mathrm{~mW} / \mathrm{cm}^{2}$ ultraviolet A (UVA) light for $30 \mathrm{~min}$. The pl-ACXL group was treated with the KXL system using $0.1 \%$ riboflavin with HPMC presoak for $10 \mathrm{~min}$, followed by $8 \mathrm{~min}$ (1 sec on/1 sec off) of $30 \mathrm{~mW} / \mathrm{cm}^{2}$ UVA light. Patients were evaluated according to the uncorrected distance visual acuity (UDVA), corrected DVA (CDVA), refraction, maximum keratometry (Kmax), endothelial cell density (ECD), anterior segment optical coherence tomography and in vivo confocal microscopy. The follow-up period was 12 months. Transient haze was observed in 17 eyes (47.22\%) in the CXL group and 8 eyes $(22.22 \%)$ in the pl-ACXL group at 1 month postoperatively. There were no significant postoperative differences in the astigmatism, manifest refraction spherical equivalent, ECD or thinnest corneal thickness. By contrast, UDVA, CDVA and Kmax presented significant improvement at 12 months postoperatively in the two groups. The demarcation line depth was $284.94 \pm 33.29 \mu \mathrm{m}$ in the CXL group, which was significantly deeper in comparison with that in the pl-ACXL group $(201.64 \pm 27.72 \mu \mathrm{m} ; \mathrm{P}<0.01)$ at 1 month postoperatively. In vivo
\end{abstract}

Correspondence to: Dr Shi-Yan Qiu, Department of Pediatrics, The People's Hospital of Linyi, 27, Jiefang Road, Linyi, Shandong 276000, P.R. China

E-mail: shiyanqiu0613@163.com

Key words: keratoconus, conventional corneal collagen cross-linking, pulsed light accelerated corneal collagen cross-linking, demarcation line, in vivo confocal microscopy confocal microscopy revealed keratocyte apoptosis and stromal edema at 1 month postoperatively, which gradually recovered towards the normal status after 12 months in the two groups. There were no apparent changes in the posterior stroma and endothelium in either group. The results of the present study revealed that CXL and pl-ACXL were safe and effective procedures in stabilizing the progression of keratoconus. The CXL technique offers more effective visual and topographic outcomes compared with pl-ACXL, while pl-ACXL ensures shorter treatment time and reduced microstructural damage.

\section{Introduction}

Keratoconus is an idiopathic degenerative eye disease characterized by a progressive non-inflammatory thinning and conical protrusion of the cornea, which results in corneal protrusion, irregular astigmatism, loss of visual acuity and even the possibility of corneal perforation (1). Keratoconus is the most prevalent form of corneal ectasia and affects all ethnicities. However, higher incidence has been reported in the Asian population when compared with Caucasian individuals $(2,3)$. Although the etiology and pathology of the disease remain not fully understood, certain studies have identified that in the process of keratoconus, the intrafibrillar and interfibrillar collagen fiber cross-links are diminished and lost due to the apoptosis of keratocytes or release of proteolytic enzymes $(4,5)$.

Corneal collagen cross-linking (CXL) with the photo-sensitizer riboflavin and ultraviolet A (UVA) light represents a landmark in the management of keratoconus since it directly targets the underlying pathology rather than only addressing the refractive consequences of the disorder $(6,7)$, due to its capacity in increasing biomechanical corneal resistance $(8,9)$ and intrinsic anti-collagenase activity (10). Conventional CXL (CXL) with a continuous irradiation of $3 \mathrm{~mW} / \mathrm{cm}^{2}$ for $30 \mathrm{~min}$ is considered safe and effective in the prevention of keratoconus progression according to different clinical trials (11-13). However, the procedure is time-consuming, lasting $\sim 1 \mathrm{~h}$, which may result in patient discomfort and reduced physician working efficiency (14). According to the Bunsen-Roscoe law of reciprocity, it is theoretically possible to deliver the 
same energy dose ensuring a proportional biological effect by setting a higher UVA power in a shorter exposure time in the accelerated CXL modality (15-17). However, a lower experimental and clinical efficacy of accelerated CXL has been reported, which was attributed to the higher consumption and shortage of oxygen in the stroma $(18,19)$. Delivering ultraviolet light with an on-off pattern is expected to allow more oxygen to diffuse into the corneal stroma, lead to an enhanced release of singlet oxygen and allow a more effective cross-linking of the collagen molecules. Considering these aforementioned factors, pulsed-light accelerated CXL (pl-ACXL) has been recognized by physicians and is currently gaining popularity. For instance, Peyman et al (20) demonstrated that the pl-ACXL protocol induced a significantly deeper stromal demarcation line when compared with the continuous accelerated CXL protocol.

In the present study, the clinical outcomes of CXL ( $3 \mathrm{~mW} / \mathrm{cm}^{2}$ for $30 \mathrm{~min}$ ) and $\mathrm{pl}$-ACXL $\left(30 \mathrm{~mW} / \mathrm{cm}^{2}\right.$ for $8 \mathrm{~min}$ with $1 \mathrm{sec}$ on/1 sec off) were evaluated in a series of 72 eyes with progressive keratoconus in 58 patients over a 12-month follow-up period. The treatment penetration was estimated by means of in vivo confocal microscopy.

\section{Materials and methods}

Subjects. This prospective study included 58 patients with 72 eyes with keratoconus who were treated between January 2015 and August 2016 in the Department of Ophthalmology, Shandong Provincial Hospital affiliated to Shandong University (Jinan, Shandong). Of these, 36 eyes of 31 patients were treated with CXL (CXL group) and 36 eyes of 27 patients were treated with pl-ACXL (pl-ACXL group). The treatment protocols of CXL were randomly selected, and all patients were followed for at least 12 months. Diagnosis of keratoconus was established by the Amsler-Krumeich classification, based on the astigmatism, corneal power, corneal transparency and corneal thickness, obtained with a rotating Scheimpflug imaging instrument (Pentacam; Oculus Optikgeräte $\mathrm{GmbH}$, Wetzlar, Germany), slit-lamp biomicroscopy (21) and interaocular pressure (IOP) using Goldmann applanation tonometry (Haag-Streit, Koniz, Switzerland). As keratoconus is characterized by binocular asymmetry, when both eyes of keratoconus patients were treated with CXL, the same CXL protocol was selected.

The inclusion criteria were as follows: Progressive keratoconus of stages 1-3 according to the Amsler-Krumeich classification $(21,22)$; the thinnest corneal thickness (TCT) was $>400 \mu \mathrm{m}$; and patients did not wear contact lenses for one month prior to the initial evaluation and treatment. Progression of the disease was considered as confirmed if the loss of corrected distance visual acuity (CDVA) was more than one line in 1 year, or when the topographic keratometry increased by $>1.0 \mathrm{D}$ in 6 months or $>2.0 \mathrm{D}$ in 12 months. Patients with ocular, corneal or immune system disorders, as well as those who were pregnant or breastfeeding, were excluded from the present study. All participants signed an informed consent form in accordance with the tenets of the Declaration of Helsinki. The present study also received Institutional Review Board approval by the Shandong Provincial Hospital affiliated to Shandong University.
CXL treatment. The CXL procedure was performed under sterile conditions. Subsequent to topical anesthesia using $0.5 \%$ proparacaine hydrochloride (Alcaine; Alcon Laboratories, Inc., Fort Worth, TX, USA) eye drops, a lid speculum was inserted. To loosen the epithelium from the stroma, the central cornea was contacted with a filter paper (diameter, $9 \mathrm{~mm}$ ) soaked with $20 \%$ alcohol for $60 \mathrm{sec}$, and then the central $9 \mathrm{~mm}$ of the cornea epithelium was removed with a blunt spatula (AE-2766; Asico LLC, Westmont, IL, USA). Deepithelialization was followed by measuring of the corneal thickness with ultrasound pachymetry (Pachy Meter SP3000; Tomey Corp., Nagoya, Japan) to validate that the TCT was $>400 \mu \mathrm{m}$. Following epithelial debridement, $0.1 \%$ riboflavin solution in $20 \%$ dextran (Shandong Fangming Pharmaceutical Group Co., Ltd., Heze, China) was applied to the cornea every $3 \mathrm{~min}$ for $30 \mathrm{~min}$. A digital slit-lamp photograph was performed to ensure the appearance of riboflavin in the anterior chamber. Next, the eye was irradiated with UVA light with a 370-nm wavelength (UVX 1000 system; IROC Innocross AG, Zurich, Switzerland) at a working distance of $5 \mathrm{~cm}$. An area with 9-mm diameter in the center of the cornea was irradiated with $3 \mathrm{~mW} / \mathrm{cm}^{2}$ for $30 \mathrm{~min}$. During the irradiation, $0.1 \%$ riboflavin solution was applied every $3 \mathrm{~min}$ to maintain the riboflavin saturation in the cornea stroma. The total exposure dose was $5.4 \mathrm{~J} / \mathrm{cm}^{2}$.

At the end of the procedure, a bandage-type corneal contact lens was applied until complete closure of the corneal epithelium was observed. Postoperative medication included a combination of $0.1 \%$ dexamethasone and $0.3 \%$ tobramycin (TobraDex; Alcon Laboratories, Inc.) four times a day, and the dose was tapered over 2 weeks.

pl-ACXL treatment. Patients were prepared using the same process as for the CXL procedure. Following epithelial debridement, $0.1 \%$ dextran-free riboflavin with hydroxyl, propyl, methyl and cellulose (VibeX Rapid; Avedro Inc., Waltham, MA, USA) were instilled every 2 for $10 \mathrm{~min}$. After riboflavin had been observed in the anterior chamber, the KXL system (Avedro Inc.) was applied to irradiate the cornea with UVA light at a $365-\mathrm{nm}$ wavelength, delivered using $30 \mathrm{~mW} / \mathrm{cm}^{2}$ irradiance. The 'pulsed-light' irradiation mode was used to alternate 1 sec of UVA irradiation with a $1 \mathrm{sec}$ pause, for a total duration of $8 \mathrm{~min}$. The cumulative dose was $7.2 \mathrm{~J} / \mathrm{cm}^{2}$. During UVA irradiation, balanced salt solution was distilled onto the subject's eyes to prevent dry spots on the surface of the cornea. Postoperatively, a bandage-type corneal contact lens was applied until complete closure of the corneal epithelium, followed by application of the same postoperative medications as those administered subsequent to the CXL procedure.

Preoperative and postoperative examination items. All patients received systematic ophthalmologic examinations preoperatively and at each follow-up visit. Examination included measurement of the uncorrected distance visual acuity (UDVA), corrected DVA (CDVA) and manifest refraction spherical equivalent (MRSE). Tomography data were recorded using Pentacam, including the maximum keratometry (Kmax) and TCT. Corneal tomographic images were obtained with optical coherence tomography (OCT) with the RTVue 
Table I. Baseline demographic and clinical characteristics of patients in the CXL and pl-ACXL groups.

\begin{tabular}{lccc}
\hline Parameter & CXL & pl-ACXL & P-value \\
\hline $\begin{array}{l}\text { No. of } \\
\text { patients (n) }\end{array}$ & 31 & 27 & - \\
$\begin{array}{l}\text { No. of eyes } \\
\text { treated (n) }\end{array}$ & 36 & 36 & - \\
$\begin{array}{l}\text { Male gender } \\
\text { (\%) }\end{array}$ & $18(58.06)$ & $13(48.15)$ & 0.45 \\
Age (years) & $26.86 \pm 5.28$ & $25.03 \pm 5.2$ & 0.142 \\
$\begin{array}{l}\text { UDVA } \\
\text { (logMAR) }\end{array}$ & $0.9 \pm 0.34$ & $0.82 \pm 0.37$ & 0.356 \\
CDVA & $0.36 \pm 0.25$ & $0.28 \pm 0.23$ & 0.159 \\
$\begin{array}{l}\text { (logMAR) } \\
\text { Astigmatism }\end{array}$ & $-3.32 \pm 1.69$ & $-2.89 \pm 1.43$ & 0.251 \\
$\begin{array}{l}\text { (D) } \\
\text { MRSE (D) }\end{array}$ & $-6.12 \pm 3.96$ & $-5.54 \pm 3.21$ & 0.496 \\
$\begin{array}{l}\text { Kmax (D) } \\
\text { TCT }(\mu \mathrm{m})\end{array}$ & $54.38 \pm 5.65$ & $53.05 \pm 4.8$ & 0.284 \\
ECD & $456.53 \pm 27.57$ & $444.22 \pm 31.81$ & 0.084 \\
$\left(\right.$ cell $\left./ \mathrm{mm}^{2}\right)$ & $2658.17 \pm 265.84$ & $2563.92 \pm 238.9$ & 0.118 \\
IOP $(\mathrm{mmHg})$ & $14.07 \pm 2.21$ & $13.54 \pm 2.12$ & 0.302 \\
\hline
\end{tabular}

CXL, conventional corneal cross-linking; pl-ACXL, pulsed light accelerated corneal cross-linking; UDVA, uncorrected distance visual acuity; CDVA, corrected distance visual acuity; MRSE, manifest refraction spherical equivalent; Kmax, maximum keratometry; TCT, thinnest corneal thickness; ECD, endothelium cell density; IOP, intraocular pressure.

OCT system (Optovue, Inc., Fremont, CA, USA). Various microstructural features of the cornea were observed with a Heidelberg Retina Tomograph confocal microscope with the Rostock Corneal Module (HRT III; Heidelberg Engineering, Inc., Heidelberg, Germany). Patients were evaluated preoperatively and at 1, 3, 6 and 12 months postoperatively. The parameters were recorded at the follow-up visits by the same experienced technician as prior to surgery.

Statistical analysis. Statistical analyses were performed using SPSS version 20 software (IBM Corp., Armonk, NY, USA). The Kolmogorov-Smirnov test was used to check for a normal distribution of quantitative data, which are provided as the mean \pm standard deviation. Postoperative changes were evaluated using a paired $t$-test. If the data were not distributed normally, the Wilcoxon rank-sum test was performed. An independent sample t-test was performed to analyze the difference in outcomes between the two groups, while the Mann-Whitney test was performed when data were not distributed normally. A P-value of $<0.05$ was considered to indicate differences that were statistically significant.

\section{Results}

Patient characteristics and baseline values. In the present study, CXL or pl-ACXL was performed on 72 eyes of
Table II. Changes in clinical characteristics of eyes in the CXL and pl-ACXL groups at 12 months postoperatively compared with the baseline measurements.

\begin{tabular}{lccc}
\hline Parameter & CXL & pl-ACXL & P-value $^{\mathrm{a}}$ \\
\hline $\begin{array}{l}\Delta \text { UDVA } \\
\text { (logMAR) }\end{array}$ & $0.14 \pm 0.05$ & $0.12 \pm 0.04$ & 0.769 \\
$\begin{array}{l}\Delta \text { CDVA } \\
\text { (logMAR) }\end{array}$ & $0.12 \pm 0.03$ & $0.09 \pm 0.02$ & 0.323 \\
$\begin{array}{l}\Delta \text { Astigmatism } \\
\text { (D) }\end{array}$ & $0.35 \pm 1.55$ & $0.45 \pm 1.34$ & 0.198 \\
$\begin{array}{l}\text { MRSE (D) } \\
\Delta \text { Kmax (D) }\end{array}$ & $0.5 \pm 1.58$ & $0.6 \pm 1.78$ & 0.189 \\
$\begin{array}{l}\Delta \mathrm{ECD} \\
\left(\mathrm{cell} / \mathrm{mm}{ }^{2}\right)\end{array}$ & $109.56 \pm 2.78$ & $1.31 \pm 2.34$ & 0.537 \\
$\Delta \mathrm{TCT}(\mu \mathrm{m})$ & $445.56 \pm 26.06$ & $440.31 \pm 32.04$ & 0.448 \\
$\Delta \mathrm{IOP}(\mathrm{mmHg})$ & $13.39 \pm 2.52$ & $13.36 \pm 1.79$ & 0.954 \\
\hline
\end{tabular}

${ }^{\mathrm{a}}$ comparing CXL and pl-ACXL groups at 12 months postoperatively. $\Delta$, changes in clinical characteristics at 12 months postoperatively compared with the baseline measurements; CXL, conventional corneal cross-linking; pl-ACXL, pulsed light accelerated corneal cross-linking; UDVA, uncorrected distance visual acuity; CDVA, corrected distance visual acuity; MRSE, manifest refraction spherical equivalent; Kmax, maximum keratometry; ECD, endothelium cell density; TCT, thinnest corneal thickness; IOP, intraocular pressure.

58 patients with progressive keratoconus. At baseline, there were no significant differences between the CXL and pl-ACXL groups in terms of their age, UDVA, CDVA, astigmatism, MRSE, Kmax, TCT, ECD or IOP values. The baseline parameters are summarized in Table I.

Postoperative complications. Following surgery, stromal haze was observed in 17 eyes (47.22\%) in the CXL group and 8 eyes $(22.22 \%)$ in the pl-ACXL group at the 1-month postoperative visit. The haze disappeared in all eyes by 12 months after the procedure. In the two groups, all patients selected presented no delayed corneal epithelium healing, corneal melting, permanent scars, endothelial damage, sterile infiltrates, corneal infection or other complications during the 12-month follow-up period.

Visual acuity and refractive outcomes. At the 12-month follow-up, UDVA demonstrated a statistically significant improvement of $0.14 \pm 0.05$ and $0.12 \pm 0.04 \log$ MAR chart scores in the CXL and pl-ACXL groups, respectively (both $\mathrm{P}<0.001$; Fig. 1A). In addition, the CDVA exhibited a statistically significant improvement of $0.12 \pm 0.03$ and $0.09 \pm 0.02$ $\operatorname{logMAR}$ in the CXL and pl-ACXL groups, respectively (both $\mathrm{P}<0.001$; Table II and Fig. 1B). However, there were no statistically significant differences in postoperative astigmatism or MRSE between the CXL and pl-ACXL groups (all P>0.05). Furthermore, no statistically significant differences were detected in the astigmatism or MRSE between the postoperative and baseline values in the two groups (all $\mathrm{P}>0.05$ ). 


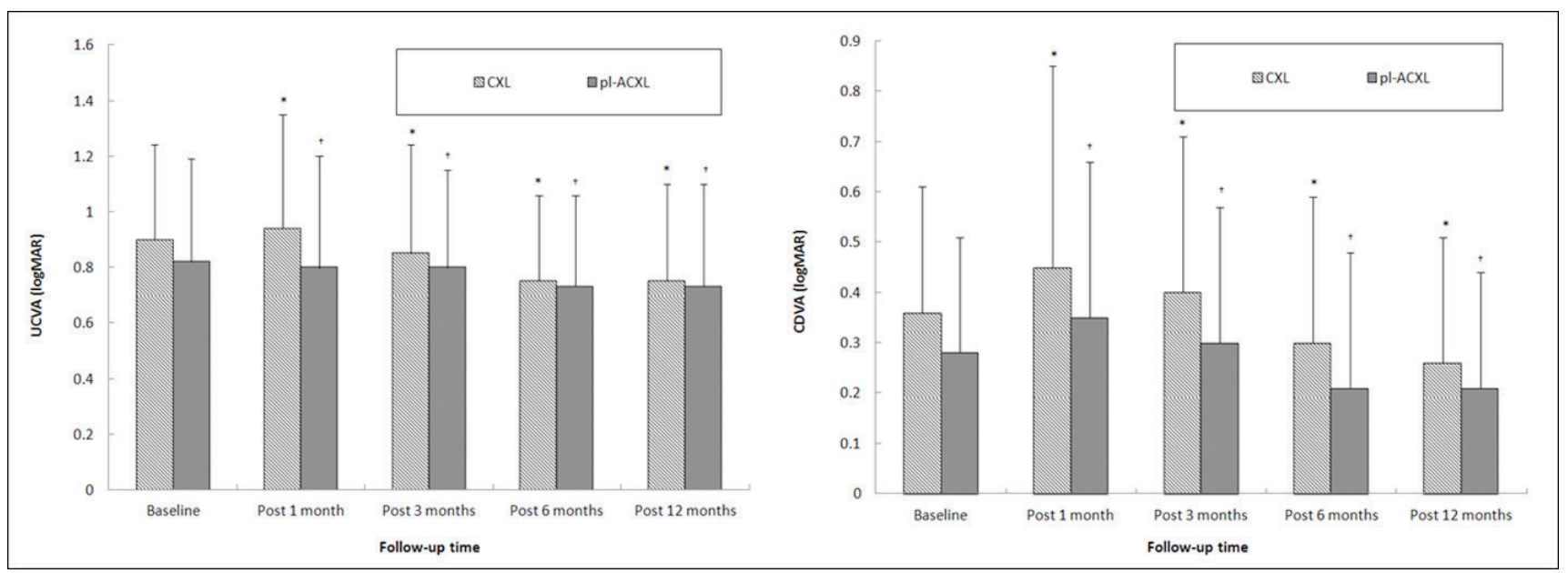

Figure 1. (A) UDVA and (B) CDVA prior to and following CXL and pl-ACXL. "P $<0.05$ vs. preoperative value in the CXL group; ${ }^{\dagger} \mathrm{P}<0.05$ vs. preoperative value in pl-ACXL group. CXL, conventional corneal collagen cross-linking; pl-ACXL, pulsed-light accelerated corneal collagen cross-linking; UDVA, uncorrected distance visual acuity; CDVA, corrected distance visual acuity.



Figure 2. Maximum keratometry values prior to and following the CXL and pl-ACXL procedures. ${ }^{*} \mathrm{P}<0.05$ vs. preoperative value in the $\mathrm{CXL}$ group; ${ }^{P}<0.05$ vs. preoperative value in pl-ACXL group. CXL, conventional corneal collagen cross-linking; pl-ACXL, pulsed-light accelerated corneal collagen cross-linking.

Topographic results. Fig. 2 demonstrates the Kmax readings from the Pentacam system preoperatively and at the 12-month follow-up visit. Following CXL treatment, the Kmax values in the pl-ACXL group initially increased at 1 and 3 months, and later decreased at 6 and 12 months. There was a notable improvement in the treated eyes, with the Kmax decreasing by $1.80 \pm 2.78 \mathrm{D}$ in the CXL group and $1.31 \pm 2.34 \mathrm{D}$ in the pl-ACXL group at 12 months post treatment compared with the baseline. In the CXL group, $94.44 \%$ of the eyes $(34 / 36)$ presented flattened or stable Kmax values, as compared with $88.89 \%$ of the eyes $(32 / 36)$ in the pl-ACXL group.

Demarcation line. A demarcation line can be observed between the anterior hyper-reflective stroma and posterior stroma with normal reflectivity. The mean stromal demarcation line depth was $284.94 \pm 33.29 \mu \mathrm{m}$ (range, $236-372 \mu \mathrm{m}$ ) in the CXL group and 201.64 $\pm 27.72 \mu \mathrm{m}$ (range, 163-279 $\mu \mathrm{m}$ ) in the pl-ACXL group. Thus, the demarcation line depth of eyes in the CXL group was deeper in comparison with that in the pl-ACXL group, with a statistically significant difference observed $(\mathrm{P}<0.001$; Fig. 3$)$.

In vivo confocal microscopy and ECD. Following CXL and pl-ACXL, in vivo confocal microscopy images demonstrated that the sub-basal nerve plexus was obliterated, and the density of the nerve plexus decreased at 1, 3 and 6 months postoperatively. However, this density returned to the preoperative status at 12 months in the two groups. In addition, in the 1-3-month postoperative period following CXL, anterior stromal edema with honeycomb-like structures appeared and the keratocyte density decreased. These changes were similar in the two treatment groups, however, they were more pronounced following the CXL procedure. At 3 months, repopulation of the anterior stroma with keratocyte 



Figure 3. The horizon meridian cross-sectional optical coherence tomography images of cornea 1 month following (A) conventional corneal collagen cross-linking and (B) pulsed-light accelerated corneal collagen cross-linking.

(A)

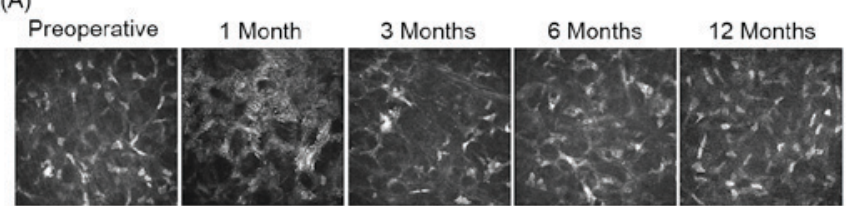

(B)

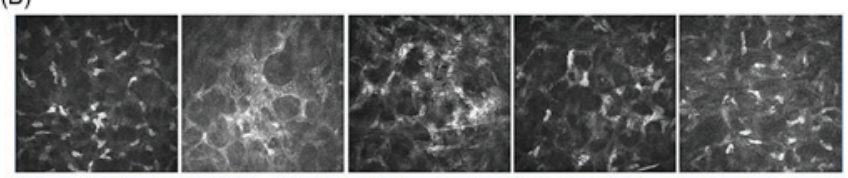

Figure 4. In vivo confocal images of the changes observed following (A) conventional corneal collagen cross-linking and (B) pulsed-light accelerated corneal collagen cross-linking at an anterior corneal depth of $\sim 150 \mu \mathrm{m}$.

nuclei was noted in CXL and pl-ACXL-treated eyes, while the honeycomb-like structures were still apparent, but less pronounced. At 12 months, the anterior stroma structure was almost restored to the preoperative status in both groups. The posterior stromal layers did not appear to have been affected in the CXL or pl-ACXL groups. Confocal images of the changes following CXL and pl-ACXL at an anterior corneal depth of $\sim 150 \mu \mathrm{m}$ are presented in Fig. 4. Furthermore, a significant difference in ECD was not observed at any of the follow-up time points when compared with the baseline value in either group.

\section{Discussion}

The efficacy of CXL, using $3 \mathrm{~mW} / \mathrm{cm}^{2}$ ultraviolet A (UVA) light for $30 \mathrm{~min}$, has been supported by various randomized controlled studies $(6,23)$. However, this protocol is time-consuming, thus research efforts are focusing on reducing the treatment duration and discomfort, as well as improving the safety of the procedure. pl-ACXL refers to a faster procedure with higher radiation and reduced exposure time to maintain a nearly constant total irradiance and efficacy, according to the photochemical law of reciprocity (16). Previous studies have demonstrated the efficacy and safety of accelerated CXL as a corneal stabilizing method for treating keratoconus $(17,24)$. In the present study a comparative analysis demonstrated the efficacy of CXL and pl-ACXL in stabilizing keratoconus progression after 12 months of follow-up, although a small case series was used. To the best of our knowledge, no previous studies exist in the literature comparing the results of CXL and $\mathrm{pl}-\mathrm{ACXL}$ procedures.

At the early stages following CXL and pl-ACXL, all patients included in the current study presented no delayed corneal epithelium healing, corneal melting, permanent scars, endothelial damage, sterile infiltrates, corneal infection or other complications. However, 17 eyes $(47.22 \%)$ in the CXL group and 8 eyes $(22.22 \%)$ in the pl-ACXL group exhibited different degrees of haze, which reached peak value at 1 month after the surgery and gradually faded away within 3-12 months after the surgery. Post-CXL corneal haze is usually a temporary and common complication, which may occur in 10-90\% of eyes treated with CXL (25). Haze may be caused by the complex structural and physiological wound-healing alterations, such as the hyperplasia of fibroblasts, in the cornea stroma following CXL. Thus, it is a distinct clinical component of the basic CXL healing process $(26,27)$. It has been demonstrated that the possibility of haze occurrence is not associated with the type of CXL surgery (14). However, in the present study, the incidence of haze in the CXL group was significantly higher in comparison with that in the pl-ACXL group $(\mathrm{P}=0.026)$. This may be due to the longer exposure time of the corneal stroma during the CXL process. The current study observed that the occurrence rate of haze following pl-ACXL was $22.22 \%$, which is in agreement with the study by Waszczykowska et al (28) reporting a $25 \%$ occurrence rate following accelerated CXL $\left(6 \mathrm{~mW} / \mathrm{cm}^{2} ; 15 \mathrm{~min}\right)$ with a 2-years follow-up. The correlation between CXL and postoperative haze has to be further analyzed through the analysis of a larger sample size.

The effects of CXL mainly present as variations of keratometry over time, as observed on corneal topography. A randomized control trial of CXL in progressive keratoconus reported that, after 36 months of follow-up, $41 \mathrm{CXL}$ treated eyes experienced a mean reduction of Kmax by $1.03 \mathrm{D}$ (6). In a study by Caporossi et al (29), with a minimum of 4-year follow-up, the mean value of Kmax decreased by $1.96 \mathrm{D}$ at 1 year postoperatively. The present study findings were similar to these aforementioned results. In the current study, a decrease of the Kmax values was observed at 12 months after the two treatments (CXL reduced by $-1.80 \pm 2.78 \mathrm{D}$; pl-ACXL reduced by $-1.31 \pm 2.34 \mathrm{D}$ ), which indicated that the corneas became flattened. Therefore, $\mathrm{pl}-\mathrm{ACXL}$ treatment appears to be as effective as CXL. In addition, the difference in treatment protocols resulted in the pl-ACXL group receiving higher total UVA irradiation $\left(7.2 \mathrm{~J} / \mathrm{cm}^{2}\right.$ at a $30 \mathrm{~mW} / \mathrm{cm}^{2}$ irradiance for $8 \mathrm{~min}$, with $1 \mathrm{sec}$ on/1 sec off) as compared with that in the CXL group $\left(5.4 \mathrm{~J} / \mathrm{cm}^{2}\right.$ with an irradiance of $3 \mathrm{~mW} / \mathrm{cm}^{2}$ over $30 \mathrm{~min}$ ). In the study by Mazzotta et al (30), which used the same pl-ACXL protocol as the present study, the apical curvature of the treated cornea demonstrated a decrease by a mean value of $1.39 \pm 0.38 \mathrm{D}$ at 12 months of follow-up. The efficacy of accelerated CXL is also supported by experimental data on 
human donor corneas with scanning acoustic microscopy. The results demonstrated an increase in stiffness of the corneal tissue by the same factor of 1.051 following treatment with the convention and accelerated CXL protocols (31).

The demarcation line represents the effective depth following CXL (32). In the present study, the interaction depth of CXL at 1 month after surgery was observed by performing anterior segment OCT. The CXL treatment presented a deeper effect, at a stromal depth of $284.94 \pm 33.29 \mu \mathrm{m}$, while pl-ACXL treatment presented a penetration of $201.64 \pm 27.72 \mu \mathrm{m}$. These findings were consistent with the findings of Mazzotta et al (30), in which the demarcation line was $200 \mu \mathrm{m}$ in depth subsequent to pl-ACXL treatment. Kohlhaas et al (33) suggested that only the anterior $200 \mu \mathrm{m}$ of the cornea is affected in keratoconus. The demarcation line depth recorded by OCT in the present study suggests that the depth of cross-linking was sufficient to reach the majority of the affected cornea.

CXL induces cell apoptosis of stromal keratocytes, and one potential risk of CXL may be the damage to the corneal endothelial cells (14). Vinciguerra et al (34) reported that, following CXL surgery, stromal cell apoptosis was detected on the corneal stroma at a depth of $\sim 320 \mu \mathrm{m}$. In the CXL procedure, the UVA irradiation energy was $5.4 \mathrm{~J} / \mathrm{cm}^{2}$, markedly lower than the threshold that may cause injuries to the corneal endothelium, lens and retina in a cornea of sufficient thickness (34). In the current study, the radiation energy delivered during pl-ACXL was $7.2 \mathrm{~J} / \mathrm{cm}^{2}$. There were no statistical differences in the ECD between the baseline and at 12 months postoperatively.

Richoz et al (18) highlighted the slow rate of replenishment of oxygen in the cornea as a potential limitation of high-radiation accelerated CXL in an in vitro porcine cornea experiment. A 1-year follow-up clinical study (30) also confirmed that oxygen represents the main driver of collagen cross-linking reaction. Pulsed-light treatment optimized intraoperative oxygen availability, thus improving the postoperative functional outcomes compared with the continuous-light treatment of accelerated CXL (30). Although in the current study the functional results at 1 year after CXL and pl-ACXL demonstrated keratoconus stability in the two groups, the functional outcomes were improved in the CXL treatment group, which also presented a deeper stromal penetration.

In vivo confocal microscopy was used to observe the microstructural changes over time following CXL treatment in the present study. Severe loss of sub-basal nerves was observed in the early postoperative period in both the CXL and pl-ACXL-treated eyes. Mechanical removal of the epithelium may be mainly responsible for the loss of corneal nerves. Previous in vivo confocal microscopy has demonstrated that CXL and pl-ACXL may cause keratocyte apoptosis in the anterior and middle stroma in the early postoperative period, while by 6 months, the keratocytes had gradually repopulated the cornea $(35,36)$, which is consistent with the results of the present study. The posterior stromal keratocyte density and the endothelial cell density were unaffected by the two types of CXL. These observations are in agreement with previous studies that generally reported that the posterior stroma and endothelium qualitatively are unaffected subsequent to CXL (37,38).
The limitations of the current study include the small number of patients in each group and the short follow-up period. The long-term effects of the two cross-linking methods require further investigation. Nevertheless, in the present study, it was observed that CXL and pl-ACXL were able to control and delay the development of keratoconus to a certain extent at an early stage following the surgery. The efficacy of these techniques needs to be investigated with mid to long-term follow-up and in a large cohort of patients.

In conclusion, CXL and pl-ACXL were safe and effective procedures for stabilizing the progression of keratoconus. The CXL technique offers more effective visual and topographic outcomes than pl-ACXL; however, pl-ACXL induces less microstructural damage. The long-term effects of both cross-linking methods require further study.

\section{Acknowledgements}

The authors would like to thank Avedro Inc. (Waltham, MA, USA) for the loan of the KXL system.

\section{References}

1. Edrington TB, Zadnik K and Barr JT: Keratoconus. Optom Clin 4: 65-73, 2004.

2. Leccisotti A, Aslanides IM, Moore JE and Shah S: Keratoconus and Keratoectasia: Advancements in diagnosis and treatment. J Ophthalmol 2012: 526058, 2012.

3. Georgiou T, Funnell CL, Cassels-Brown A and O'Conor R: Influence of ethnic origin on the incidence of keratoconus and associated atopic disease in Asians and white patients. Eye 18: 379-383, 2004.

4. Meek KM, Tuft SJ, Huang Y, Gill PS, Hayes S, Newton RH and Bron AJ: Changes in collagen orientation and distribution in keratoconus corneas. Invest Ophthalmol Vis Sci 46: 1948-1956, 2005.

5. Roberts CJ and Dupps WJ Jr: Biomechanics of corneal ectasia and biomechanical treatments. J Cataract Refract Surg 40: 991-998, 2014

6. Wittig-Silva C, Chan E, Islam FM, Wu T, Whiting M and Snibson GR: A randomized, controlled trial of corneal collagen cross-linking in progressive keratoconus: Three-year results. Ophthalmology 121: 812-821, 2014.

7. Meiri Z, Keren S, Rosenblatt A, Sarig T, Shenhav L and Varssano D: Efficacy of corneal collagen cross-linking for the treatment of keratoconus: A systematic review and meta-analysis. Cornea 35: 2016

8. Kling S, Remon L, Pérez-Escudero A, Merayo-Lloves J and Marcos S: Corneal biomechanical changes after collagen cross-linking from porcine eye inflation experiments. Invest Ophthalmol Vis Sci 51: 3961-3968, 2010.

9. Wollensak G, Spoerl E and Seiler T: Stress-strain measurements of human and porcine corneas after riboflavin-ultraviolet-A-induced cross-linking. J Cataract Refract Surg 29: 1780-1785, 2003.

10. Spoerl E, Wollensak G and Seiler T: Increased resistance of crosslinked cornea against enzymatic digestion. Curr Eye Res 29: 35-40, 2004.

11. Raiskup-Wolf F, Hoyer A, Spoerl E and Pillunat LE: Collagen crosslinking with riboflavin and ultraviolet-A light in keratoconus: Long-term results. J Cataract Refract Surg 34: 796-801, 2008.

12. Hersh PS, Greenstein SA and Fry KL: Corneal collagen crosslinking for keratoconus and corneal ectasia: One-year results. J Cataract Refract Surg 37: 149-160, 2011.

13. Hashemi H, Seyedian MA, Miraftab M, Fotouhi A and Asgari S: Corneal collagen cross-linking with riboflavin and ultraviolet a irradiation for keratoconus: Long-term results. Ophthalmology 120: 1515-1520, 2013.

14. Spoerl E, Mrochen M, Sliney D, Trokel S and Seiler T: Safety of UVA-riboflavin cross-linking of the cornea. Cornea 26: 385-389, 2007. 
15. Wernli J, Schumacher S, Spoerl E and Mrochen M: The efficacy of corneal cross-linking shows a sudden decrease with very high intensity UV light and short treatment time. Invest Ophthalmol Vis Sci 54: 1176-1180, 2013.

16. Mrochen M: Current status of accelerated corneal cross-linking. Indian J Ophthalmol 61: 428-429, 2013.

17. Kurt T, Ozgurhan EB, Yildirim Y, Akcay BI, Cosar MG Bozkurt E and Taskapili M: Accelerated (18 mW/cm(2)) corneal cross-linking for progressive keratoconus: 18-Month results. J Ocul Pharmacol Ther 32: 186-191, 2016.

18. Richoz O, Hammer A, Tabibian D, Gatzioufas Z and Hafezi F: The Biomechanical effect of corneal collagen cross-linking (CXL) with riboflavin and UV-A is oxygen dependent. Transl Vis Sci Technol 2: 6, 2013.

19. Hammer A, Richoz O, Arba Mosquera S, Tabibian D, Hoogewoud F and Hafezi F: Corneal biomechanical properties at different corneal cross-linking (CXL) irradiances. Invest Ophthalmol Vis Sci 55: 2881-2884, 2014.

20. Peyman A, Nouralishahi A, Hafezi F, Kling S and Peyman M: Stromal demarcation line in pulsed versus continuous light accelerated corneal cross-linking for keratoconus. J Refract Surg 32: 206-208, 2016.

21. Kamiya K, Ishii R, Shimizu K and Igarashi A: Evaluation of corneal elevation, pachymetry and keratometry in keratoconic eyes with respect to the stage of Amsler-Krumeich classification. Br J Ophthalmol 98: 459-463, 2014.

22. Krumeich JH and Kezirian GM: Circular keratotomy to reduce astigmatism and improve vision in stage I and II keratoconus. J Refract Surg 25: 357-365, 2009.

23. O'Brart DP, Chan E, Samaras K, Patel P and Shah SP: A randomised, prospective study to investigate the efficacy of riboflavin/ultraviolet A $(370 \mathrm{~nm})$ corneal collagen cross-linkage to halt the progression of keratoconus. $\mathrm{Br} \mathrm{J}$ Ophthalmol 95 : $1519-1524,2011$.

24. Konstantopoulos A and Mehta JS: Conventional versus accelerated collagen cross-linking for keratoconus. Eye Contact Lens 41 : 65-71, 2015.

25. Razmjoo H, Rahimi B, Kharraji M, Koosha N and Peyman A: Corneal haze and visual outcome after collagen crosslinking for keratoconus: A comparison between total epithelium off and partial epithelial removal methods. Adv Biomed Res 3: 221, 2014.

26. Salomão MQ, Chaurasia SS, Sinha-Roy A, Ambrósio R Jr, Esposito A, Sepulveda R, Agrawal V and Wilson SE: Corneal wound healing after ultraviolet-A/riboflavin collagen cross-linking: a rabbit study. J Refract Surg 27: 401-407, 2011.

27. Kymionis GD, Portaliou DM, Diakonis VF, Kontadakis GA Krasia MS, Papadiamantis AG, Coskunseven E and Pallikaris AI: Posterior linear stromal haze formation after simultaneous photorefractive keratectomy followed by corneal collagen cross-linking. Invest Ophthalmol Vis Sci 51: 5030-5033, 2010.
28. Waszczykowska A and Jurowski P: Two-year accelerated corneal cross-linking outcome in patients with progressive keratoconus. Biomed Res Int 2015: 325157, 2015.

29. Caporossi A, Mazzotta C, Baiocchi S and Caporossi T: Long-term results of riboflavin ultraviolet a corneal collagen cross-linking for keratoconus in Italy: The Siena eye cross study. Am J Ophthalmol 149: 585-593, 2010.

30. Mazzotta C, Traversi C, Paradiso AL, Latronico ME and Rechichi M: Pulsed light accelerated crosslinking versus continuous light accelerated crosslinking: One-year results. J Ophthalmol 2014: 604731, 2014.

31. Beshtawi IM, Akhtar R, Hillarby MC, O'Donnell C, Zhao X, Brahma A, Carley F, Derby B and Radhakrishnan $\mathrm{H}$ : Biomechanical properties of human corneas following low- and high-intensity collagen cross-linking determined with scanning acoustic microscopy. Invest Ophthalmol Vis Sci 54: 5273-5280, 2013.

32. Seiler T and Hafezi F: Corneal cross-linking-induced stromal demarcation line. Cornea 25: 1057-1059, 2006.

33. Kohlhaas M, Spoerl E, Schilde T, Unger G, Wittig C and Pillunat LE: Biomechanical evidence of the distribution of cross-links in corneas treated with riboflavin and ultraviolet A light. J Cataract Refract Surg 32: 279-283, 2006.

34. Vinciguerra P, Camesasca FI, Albè E and Trazza S: Corneal collagen cross-linking for ectasia after excimer laser refractive surgery: 1-year results. J Refract Surg 26: 486-497, 2010.

35. Mazzotta C, Balestrazzi A, Traversi C, Baiocchi S, Caporossi T, Tommasi $\mathrm{C}$ and Caporossi A: Treatment of progressive keratoconus by riboflavin-UVA-induced cross-linking of corneal collagen: Ultrastructural analysis by Heidelberg Retinal Tomograph II in vivo confocal microscopy in humans. Cornea 26: 390-397, 2007.

36. Jordan C, Patel DV, Abeysekera N and Mcghee CN: In vivo confocal microscopy analyses of corneal microstructural changes in a prospective study of collagen cross-linking in keratoconus. Ophthalmology 121: 469-474, 2014.

37. Ku JY, Niederer RL, Patel DV, Sherwin T and Mcghee CN: Laser scanning in vivo confocal analysis of keratocyte density in keratoconus. Ophthalmology 115: 845-850, 2008.

38. Messmer EM, Meyer P, Herwig MC, Loeffler KU, Schirra F, Seitz B, Thiel M, Reinhard T, Kampik A and Auw-Haedrich C: Morphological and immunohistochemical changes after corneal cross-linking. Cornea 32: 111-117, 2013. 